\title{
Probing exotic magnetic phases in ferrites with neutrons
}

\author{
L MADHAV RAO
}

Solid State Physics Division. Bhabha Atomic Research Centre. Bombay 400085, India

\begin{abstract}
The decades of the sixties and early seventies saw the neutron diffraction technique playing a major role in the elucidation of magnetic structures in a variety of ferrites in our laboratory. In 1979. Villain in his seminal paper on insulating spin glasses argued that the spinel structure affords topological frustration which can give rise to a variety of perturbed magnetic ordering, depending upon magnetic dilution in the ferrite. The neutron is a unique probe to explore the nature of spatial correlations of magnetic moments in such systems. This paper describes the exciting work carried out at Trombay recently in mixed ferrites which have led to the discovery of exotic magnetic phases like the uniaxial random ferrimagnetic phase and the canted random ferrimagnetic phase involving the coexistence of long-range magnetic order and disorder.
\end{abstract}

Keywords. Neutrons; mixed ferrites; exotic magnetic phases; ferrimagnetic phase; random magnet alloy; insulating spin glass-spinels; site percolation.

\section{Introduction}

Soft ferrites belong to the cubic spinel structure (space group $O_{h}^{7}-\mathrm{F} 3 \mathrm{dm}$ ) in which the magnetic ions occupy two inequivalent sites with tetrahedral $(A)$ and octahedral $(B)$ oxygen coordination (see figure 1). The magnetic structure of such crystals essentially depends on the type of magnetic ions residing on the $A$ and $B$ sites and on the relative strengths of the inter $\left(J_{A B}\right)$ and intra sublattice exchange interactions $\left(J_{A A}, J_{B B}\right)$. Neutron diffraction offers several advantages in the determination of the magnetic properties of spinel ferrites. The marked difference in nuclear scattering amplitudes of cations and the interaction of their magnetic moments with the neutron magnetic moment enable accurate determination of the cation distributions as well as detailed magnetic structure. The neutron diffraction technique therefore began to play a vital role from the late fifties in the elucidation of the magnetic structure of a variety of spinels. At Trombay, such studies were initiated in mid-sixties. The polarized neutron technique was exploited in full measure in this laboratory to enhance the quality of diffraction data, thus enabling one to unambiguously determine the magnetic structure of those spinels having more than one magnetic species (Madhav Rao 1985).

\section{Magnetic ordering}

As remarked earlier, the magnetic structure of the spinel depends upon the relative values of $J_{A B}, J_{B B}$ and $J_{A A}$ and in general all these interactions are negative (antiferromagnetic) with $\left|J_{A B}\right| \gg\left|J_{B B}\right|>\left|J_{A A}\right|$. In such situations, collinear or Néel type of ordering is obtained.

Yafet and Kittel (1952) theoretically considered the stability of the ground state of magnetic ordering, taking all the three exchange interactions into account and 


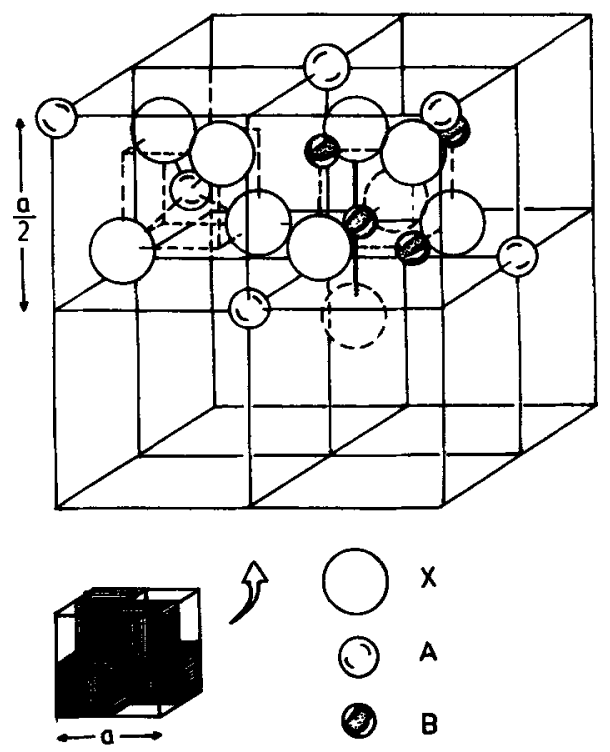

Figare 1. Primitive unit cell of the spinel structure.

concluded that beyond a certain value of $J_{B B} / J_{A B}$, the stable structure was a noncollinear triangular configuration of moments wherein the $B$ site moments are oppositely canted relative to the $A$ site moments. Later, Lyons et al (1962) extending these theoretical considerations showed that for a normal spinel the lowest energy corresponds to a conical spiral structure for the value of $3 J_{B B} / 2 J_{A B} S_{A}$ greater than unity, $S_{B}$ and $S_{A}$ being the spins or moments on the $B$ and $A$ sites respectively. Intuitively one can understand why the collinear Néel structure gets perturbed when $J_{B B} / J_{A B}$ increases. Since all these three exchange interactions are negative (favouring antiferromagnetic alignment of moments) the inter and the intra sublattice exchange interactions compete with each other in aligning the moment direction in the sublattice. This is one of the origins of topological frustration in the spinel lattice which we shall touch upon later. By selective magnetic dilution of say the $A$ sublattice one can effectively decrease the influence of $J_{A B}$ vis-a-vis $J_{B B}$ and thus perturb the Néel ordering. The first neutron diffraction study of such a system i.e. $\mathrm{Zn}_{x} \mathrm{Ni}_{1-x} \mathrm{Fe}_{2} \mathrm{O}_{4}$ was done at Trombay (Satya Murthy et al 1969) and it was shown to have the Y-K type of magnetic ordering followed by Néel ordering before passing on to the paramagnetic phase (figure 2). From our neutron study on a similar system namely $\mathrm{Zn}_{x} \mathrm{Co}_{1-x} \mathrm{Fe}_{2} \mathrm{O}_{4}$, on the other hand, we had concluded that this spinel has the $Y-K$ type of ordering right up to $T_{N}$. It must be observed that the $\mathrm{Y}-\mathrm{K}$ or conical spiral ordering truly reflects the three-dimensional long-range correlations of the spins. The question then arises: can there truly exist a three-dimensional long-range order in systems having sufficiently strong magnetic dilution, a consequence of which is to create on a microscopic scale magnetic inhomogeneities arising out of magnetic atom concentration fluctuations which in principle can lead to the existence of islands of disorder coexisting with longrange magnetic ordering? What then is the role played by these disordered clusters 


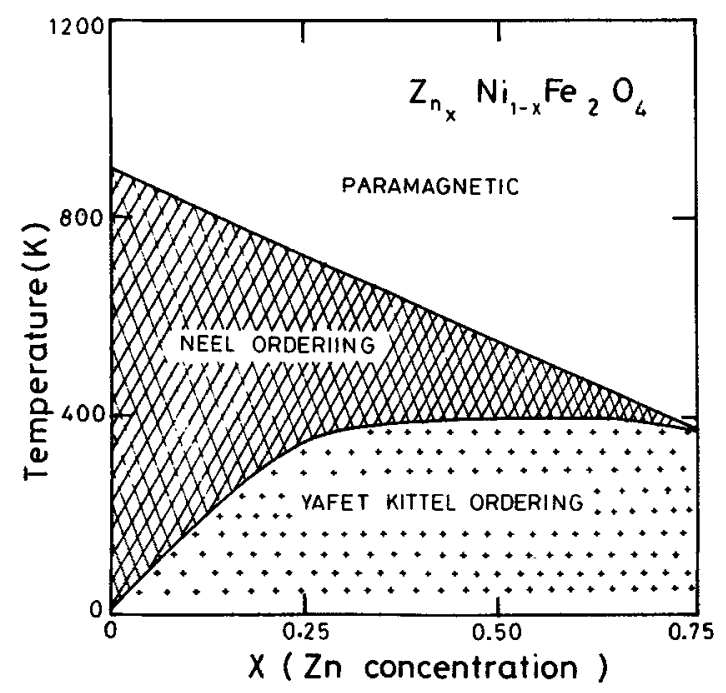

Figure 2. Magnetic phase diagram of the $\mathrm{Zn}_{x} \mathrm{Ni}_{1-x} \mathrm{Fe}_{2} \mathrm{O}_{4}$ system determined by neutron diffraction.

on the overall magnetic ordering in the system? The discovery of spin glasses in 1971 gave a fresh impetus to the study of this problem.

\section{Spin glass}

A spin glass is an unusual form of a disordered magnet. From the experimental point of view spin glasses are characterized by the presence of a low-temperature phase in which the relaxation time for attaining equilibrium is very large (as in real glasses). The susceptibility in the presence of a time-dependent magnetic field shows a 'cusp' at the so-called spin glass freezing temperature $T_{\mathrm{SG}}$ below which the low temperature phase exists. From a theoretical point of view, these properties may be connected to the practical impossibility of finding the 'true' ground state of a spin glass. Exhaustive numerical simulations on small systems show that different energy minima have different macroscopic spin configurations and the energy difference between these configurations is of the order of zero even when the volume tends to infinity. This is an unusual situation in statistical mechanics: macroscopically different configurations with the same total free energy are normally present at the point where a first-order phase transition occurs, which is an isolated point. For spin glasses this phenomenon is present in a large range of temperature and magnetic fields where no first order phase transition is seen from the thermodynamical point of view.

The origin of this peculiar behaviour is the presence of "frustration". Frustration is the inability of a spin to satisfy simultaneously all the exchange bonds to which it is subjected. This can arise, for instance, when both ferromagnetic and antiferromagnetic bonds are present. In dilute magnetic alloys (e.g. AuFe) this can arise due to random placements of the magnetic atoms in the non-magnetic host matrix and the oscillatory (RKKY) nature of the exchange interactions. In insulating systems such as in disordered spinels, this can arise in the presence of competing antiferro- 
magnetic interactions. Different terms in the Hamiltonian push in different directions and the number of possible compromises is very large. This is best illustrated in the case of an Ising Hamiltonian:

$$
\mathscr{H}=-\frac{1}{2} \sum_{i k} J_{i k} \sigma_{i} \sigma_{k}-h \sum_{i} \sigma_{i}
$$

where $h$ is the external field and $J$ the couplings between the spins $\sigma$ 's. It is clear that if the product $J_{12} J_{23} J_{31} \ldots$ is negative, it is impossible to find configurations of $J$ 's such that $\sigma_{1} J_{12} \sigma_{2}, \sigma_{2} J_{23} \sigma_{3}, \sigma_{3} J_{31} \sigma_{1} \ldots$ are all positive. It must be observed however that while frustration is an essential ingredient of a spin glass, its presence does not always lead to a disordered magnetic state. A good example of a nondisordered frustrated systems is $\mathrm{ZnCr}_{2} \mathrm{~S}_{4}$ where the coexistence of three modulated magnetic phases were seen by neutron diffraction (Hamedoun et al 1986).

\section{Insulating spin glass-spinels}

The concept of an insulating spin glass with competing antiferromagnetic interactions was developed in great detail by Villain (1979) for a three-dimensional classical spin system described by a Heisenberg Hamiltonian. Of particular interest for discussion here is the case of the cubic spinel lattice. As mentioned earlier, frustration is inherent in the spinel lattice arising from the competition between the inter and intra sublattice exchange interactions even though both these are antiferromagnetic. Frustration is also inherent in the octahedral sublattice where the $B$ sites form tetrahedra, two tetrahedra sharing a common site (figure 3). The character of the frustration of this sublattice with only nearest neighbour antiferromagnetic interactions is brought out clearly in figure 4. Many configurations having the same energy are possible, the ground state being determined by the condition that all tetrahedra have their energy separately minimized. Such a situation leads to a high ground state degeneracy preventing the establishment of long-range order. Villain (1979) showed that the ground-state degeneracy can be reduced by introducing defects such as non-magnetic impurities in the two sublattices and stabilize the spin glass phase. He proposed a magnetic phase diagram (as a function of the fractional magnetic concentrations $C_{B}$

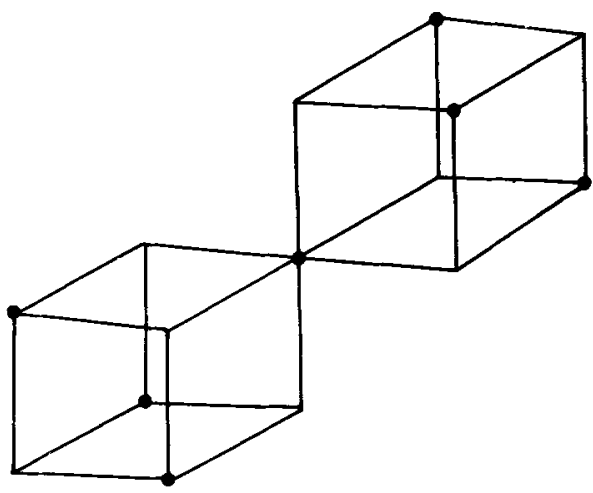

Figure 3. Octahedral sites of the spinel lattice with first nearest neighbours. 


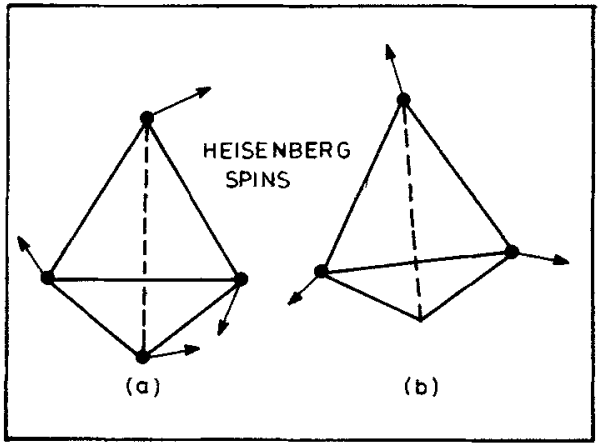

Figure 4. Ground state spin configuration of an isolated tetrahedron for a Heisenberg spin system with antiferromagnetic bonds. (a) All sites magnetic and (b) with one site substituted by a non-magnetic atom.

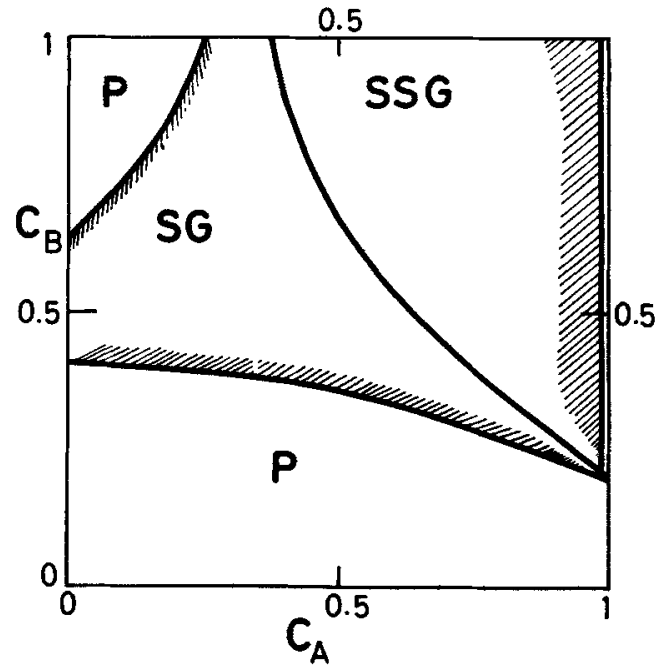

Figure 5. Villain's phase diagram of a spinel at zero temperature.

and $C_{B}$ on the $A$ and $B$ sites) (figure 5). As seen from this figure an interesting portion of the phase diagram is the semi-spin glass phase which Villain defines as a phase where the longitudinal components of the moment exhibit standard long-range ferro, ferri or antiferromagnetic order but the transverse components show spin glass order. This semi-spin glass phase shares some of the properties of a spin glass. Poole and Farach (1982) modified this phase diagram (see figure 6) taking account of the role of the $J_{A A}$ interaction which Villain had ignored.

\section{Random magnet alloy}

About the same time when Villain proposed his magnetic phase diagram for a spinel, Mookerjee (1980) established a phase diagram for a random magnetic alloy using a 
mean field effective medium theoretical approach. He analysed a classical Heisenberg system taking account of RKKY type of interactions between sites randomly occupied by magnetic atoms. The possible phases are described in figure 7 at various temperatures and magnetic atom concentration. Of particular interest (apart from the pure spin glass phase) are the uniaxial random ferro (ferri, antiferro) magnetic (URF) and the canted random ferro (ferri, antiferro) magnetic (CRF) phases. The URF phase is an unusual magnetic phase, characterized by a long-range order of the longitudinal components of the spins (or magnetic moments) with the transverse spin components being completely disordered. The CRF phase, on the other hand, continues to exhibit long-range order of the longitudinal spin components but with the transverse

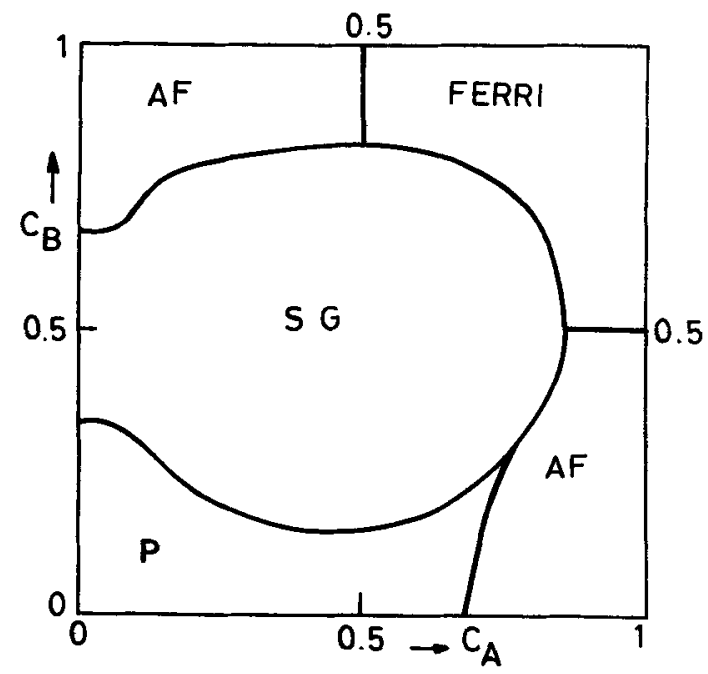

Figure 6. Poole and Farach phase diagram of a spinel at zero temperature.

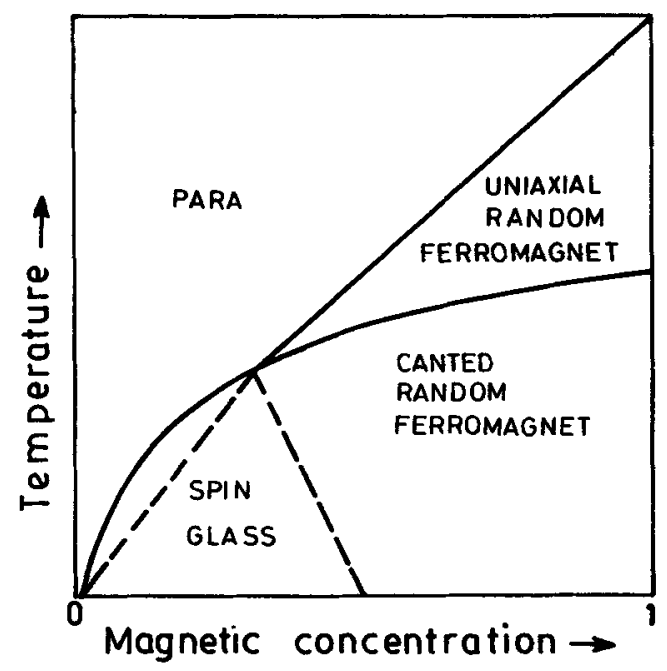

Figure 7. Mookerjee's phase diagram for a random magnetic Heisenberg alloy. 
components now frozen in random orientations. The CRF phase, in this sense, is equivalent to the semi-spin glass phase envisaged by Villain. Mookerjee's phase diagram though established for a random magnetic alloy is nevertheless relevant to the theme of this paper, since the essential principle of randomness and competing interactions which give rise to perturbed phases is the same in alloys and insulators, though the origin of these interactions differs. It is pertinent to point out here that Mookerjee's unusual magnetic phase diagram in the temperature vs magnetic atom concentration plane was published a year earlier than the oft-quoted Gabay-Toulouse model (Gabay and Toulouse 1981) which also predicts similar perturbed ordered phases.

\section{Site percolation in spinels}

The problem of site percolation in spinel sublattices was investigated by Scholl and Binder (1980) who improved upon similar attempts made earlier (Fiorani et al 1979). They established that the percolation threshold (the minimum fractional concentration of magnetic atoms needed to maintain long-range magnetic order) for the $A$ sublattice to be 0.429 and for the $B$ sublattice to be 0.390 . This approach is essentially geometrical and does not address itself to concepts such as frustration etc but nonetheless serves as a useful guide to experimenters.

Since the spinel lattice lends itself to selective magnetic dilution in both the sublattices, it is clear that the magnetic phase diagram of Villain can be explored experimentally using a variety of probes such as ac susceptibility, the Mössbauer and the neutron. In this paper, we survey the neutron work done by our group at Trombay which led to the discovery of interesting perturbed ordered phases in a series of mixed spinels.

\section{7. $\mathrm{Zn}_{x} \mathrm{Co}_{1-x} \mathrm{FeCrO}_{4}$}

A magnetic phase diagram was proposed for the disordered spinel $\mathrm{Zn}_{x} \mathrm{Co}_{1-x} \mathrm{FeCrO}_{4}$ $(x=0.0,0.2,0.4,0.5$ and 0.8$)$ on the basis of the results of the ac susceptibility, low field dc magnetization and Mössbauer studies (Muraleedharan et al 1985). This study concluded that for $x>0.4$, the system is a cluster spin glass with no long-range magnetic ordering. However, the neutron diffraction technique alone can give unambiguous microscopic evidence of the absence or presence of long-range magnetic ordering. We therefore decided to explore with neutrons this spinel system for three different $\mathrm{Zn}$ concentrations $(x=0.45,0.50$ and 0.55$)$, the magnetic ion concentration $c$ for these compositions being near the $A$ site percolation threshold $\left(c_{p}=0.429\right)$ with $c>c_{p}$. We also performed ac susceptibility measurements on these three compositions.

\subsection{Sample preparation}

The ferrite samples were prepared by the wet chemical process. This route ensures homogeneity for compositionally disordered compounds unlike the conventional ceramic method. The high purity metals were taken in appropriate ratios and dissolved in concentrated $\mathrm{HCl}$. The solution was diluted and reacted with $\mathrm{NH}_{4} \mathrm{OH}$ with constant 
stirring. To ensure the stoichiometric compositions of the metal ions, the hydroxide slurry so prepared was dried by slow heating without any filtration process.

The resulting fine mixed oxide powder was thoroughly ground and heated at $1050^{\circ} \mathrm{C}$ for a few hours followed by slow cooling. The samples so prepared were again compacted and heated at $1150^{\circ} \mathrm{C}$ for $16 \mathrm{~h}$. The powder $\mathrm{X}$-ray diffraction patterns showed a very well-defined homogeneous spinel phase formation. Room temperature ${ }^{57} \mathrm{Fe}$ Mössbauer spectra of these specimens showed that all the $\mathrm{Fe}$ ions are in the trivalent state.

\subsection{Experimental}

Neutron diffraction patterns of all the samples were measured down to $100 \mathrm{~K}$ on the linear position-sensitive detector-based powder diffractometer at the Dhruva reactor at Trombay. The $Q$ resolution of this instrument is $\sim 1.3 \%$. Neutron diffraction patterns on the $x=0.50$ specimen were measured down to $15 \mathrm{~K}$ on the liquid and amorphous diffractometer (LAD) and down to $6 \mathrm{~K}$ on the polarized neutron spectrometer (POLARIS) in the unpolarized mode at the pulsed neutron source ISIS at the Rutherford Appleton Laboratory, RAL at UK. In each case the temperature could be controlled to within $1 \mathrm{~K}$. The $Q$ resolution of LAD for the back-scattering bank of detectors is $\sim 0.4 \%$ and for the intermediate bank of detectors it is $\sim 2 \%$. The resolution of POLARIS for the intermediate bank of detectors is $\sim 4 \%$.

Low field ac susceptibility $(h=0.7 \mathrm{Oe})$ was measured on small portions of these specimens down to $4 \mathrm{~K}$, using the facility at Imperial College (UK).

\subsection{Results}

7.3a Susceptibility measurements: Temperature dependence of $\chi_{\mathrm{ac}}$ for all three compositions is shown in figure 8. For $x>0.50$, a broad distribution is seen centred around $140 \mathrm{~K}$ and at a considerably higher temperature of about $280 \mathrm{~K}$ for the $x=0.45$

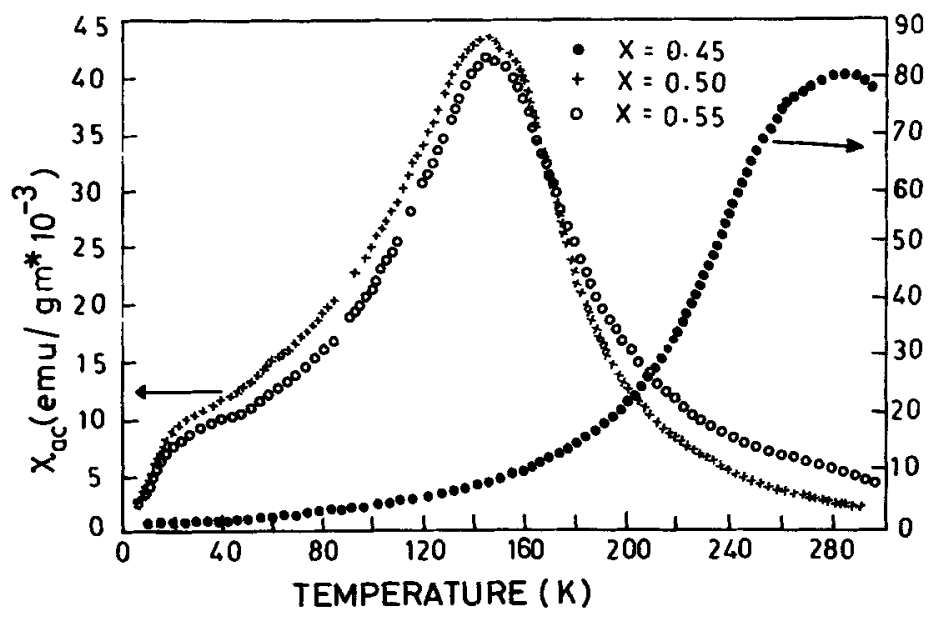

Figure 8. Temperature dependence of ac susceptibility in $\mathrm{Zn}_{x} \mathrm{Co}_{1-x} \mathrm{FeCrO}_{4}$. 
system. Low temperature response for $x=0.45$ is distinctly different from those of the $x>0.50$ systems. For $x>0.50$ specimens, a shoulder appears around $20 \mathrm{~K}$, whereas $\chi_{\mathrm{ac}}$ decreases smoothly to zero for the $x=0.45$ specimen. Furthermore, the absolute value of the susceptibility decreases drastically to almost half the value for a slight increase of the $\mathrm{Zn}$ concentration from 0.45 to 0.50 . A change of the $\mathrm{Zn}$ concentration from 0.50 to 0.55 hardly changes the shape and value of $\chi_{\mathrm{ac}}$.

7.3b Neutron diffraction measurements: In order to obtain the magnetic structure accurately it is essential to find the distribution of magnetic ions in the tetrahedral $(A)$ and octahedral $(B)$ sublattices. The neutron diffraction data taken at ambient temperature limited to the higher $Q$ region (where magnetic contributions are quite negligible), were profile-analysed using the Rietveld technique. For this system under study with a large substitutional disorder, the occupancies of some of the cations were fixed in the refinement, depending upon their site preference. It is well known that $\mathrm{Zn}$ and $\mathrm{Cr}$ have strong preferences for the $A$ site and $B$ site respectively. In the refinement, therefore, all the $\mathrm{Zn}$ ions were put on the $A$ site and all the $\mathrm{Cr}$ ions put on the $B$ sites.

The oxygen parameter $u$, the isotropic temperature factor and the lattice constant were varied as free parameters. The site occupancies of $\mathrm{Co}$ and $\mathrm{Fe}$ were varied within the constraint of satisfying the stoichiometric composition. The weighted profile $R$ factor was better than $5 \%$. The fitted profile for one of the compositions $x=0.50$ is shown in figure 9. The cation distributions obtained are summarized in table 1.

For all three compositions, it was observed that, as one lowered the temperature, the intensities of the inner Bragg reflections, namely (111), (220), (222) and (331) increased significantly, indicating important magnetic contributions to these reflections. By way of illustration, figure 10 shows the temperature evolution of the diffraction pattern taken on the $x=0.45$ specimen. It is significant to note that, within the instrumental resolution, the Bragg peaks remain sharp all through this temperature range, heralding the existence of long-range magnetic ordering. The magnetic intensities in these Bragg reflections at each temperature were separated out by the usual procedure and analysed to arrive at the magnetic structure factors and thence obtain

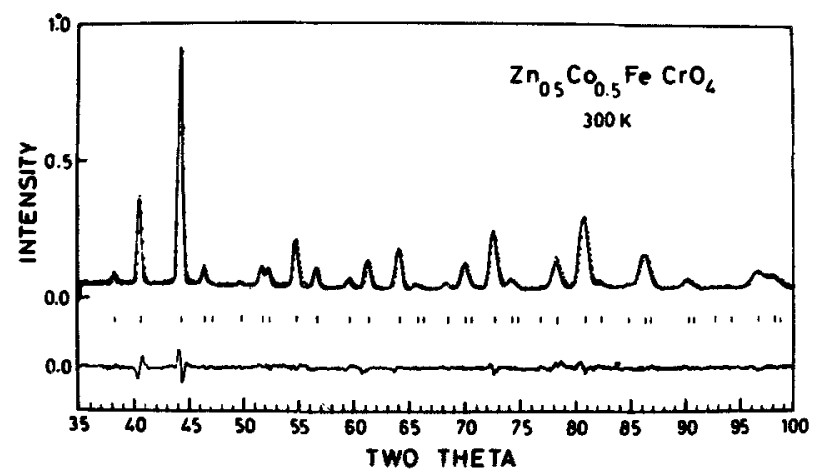

Figure 9. Neutron powder diffraction pattern of $\mathrm{Zn}_{0.5} \mathrm{Co}_{0.5} \mathrm{FeCrO}_{4}$ measured at $\lambda=1 \cdot 10 \AA$. Solid line is the calculated profile and dots are experimental points. The difference profile is also shown. 
Table 1. Summary of the neutron diffraction results in $\mathrm{Zn}_{x} \mathrm{Co}_{1-x} \mathrm{FeCrO}_{4}$ $(x=0.45,0.50$ and 0.55$)$.

\begin{tabular}{|c|c|c|c|c|c|}
\hline \multirow[b]{3}{*}{$x$} & \multirow[b]{3}{*}{$\begin{array}{l}\text { Cation } \\
\text { distribution }\end{array}$} & \multicolumn{4}{|c|}{ Site moment $\left(\mu_{B}\right)$ at $100 \mathrm{~K}$} \\
\hline & & \multicolumn{2}{|c|}{$A$ site } & \multicolumn{2}{|c|}{$B$ site } \\
\hline & & Expt. & $\begin{array}{l}\text { Free } \\
\text { ion }\end{array}$ & Expt. & $\begin{array}{l}\text { Free } \\
\text { ion }\end{array}$ \\
\hline 0.45 & $\begin{array}{c}\left(\mathrm{Zn}_{0.45} \mathrm{Co}_{0.43} \mathrm{Fe}_{0.12}\right)_{A} \\
{\left[\mathrm{Co}_{0.12} \mathrm{Fe}_{0.88} \mathrm{Cr}\right]_{B}}\end{array}$ & $2 \cdot 1(1)$ & $1 \cdot 87(2)$ & $1 \cdot 6(1)$ & $3.84(2)$ \\
\hline 0.50 & $\begin{array}{c}\left(\mathrm{Zn}_{0.30} \mathrm{Co}_{0.42} \mathrm{Fe}_{0.08}\right)_{A} \\
{\left[\mathrm{Fe}_{0.92} \mathrm{Co}_{0.08} \mathrm{Cr}\right]_{B}}\end{array}$ & $1 \cdot 3(1)$ & $1 \cdot 64(2)$ & $1.4(2)$ & $3 \cdot 88(2)$ \\
\hline 0.55 & $\begin{array}{c}\left(\mathrm{Zn}_{0.55} \mathrm{Co}_{0.38} \mathrm{Fe}_{0.07}\right)_{A} \\
{\left[\mathrm{Fe}_{0.93} \mathrm{Co}_{0.07} \mathrm{Cr}\right]_{B}}\end{array}$ & $1 \cdot 1(2)$ & $1 \cdot 37(2)$ & $1 \cdot 0(2)$ & $3 \cdot 50(2)$ \\
\hline
\end{tabular}

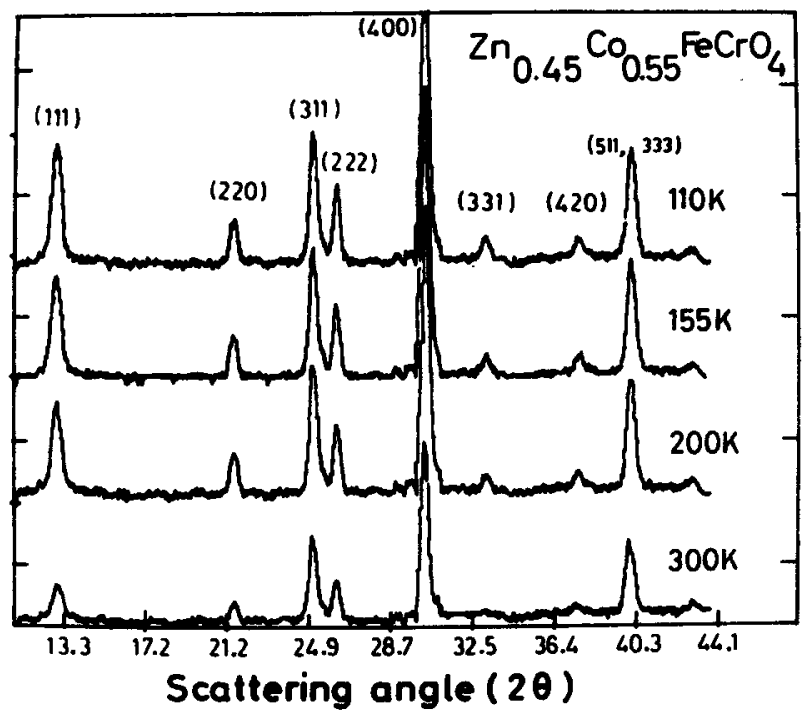

Figure 10. The temperature evolution of the diffraction pattern for $\mathrm{Zn}_{0.45} \mathrm{Co}_{0.55} \mathrm{FeCrO}_{4}$.

the ordered magnetic moments on the $A$ and $B$ sites. The ordered magnetic moments on the $A$ and $B$ sites contribute in different proportions to the magnetic structure factors of (111) and (331). The (220) and (222) magnetic structure factors, on the other hand, uniquely reflect the $A$ and $B$ site-ordered moments respectively.

The temperature variation of the (111) magnetic structure factor for all the three compositions is shown in figure 11. This figure reveals essentially two interesting features. First, the ordered moment for the $x=0.45$ specimen is significantly larger than that for the other two compositions. Secondly, the temperature variation for $x=0.45$ is smooth while those of $x=0.50$ and 0.55 show slope changes. These steplike features are brought out more prominently in figure 12 for the $x=0.50$ sample 


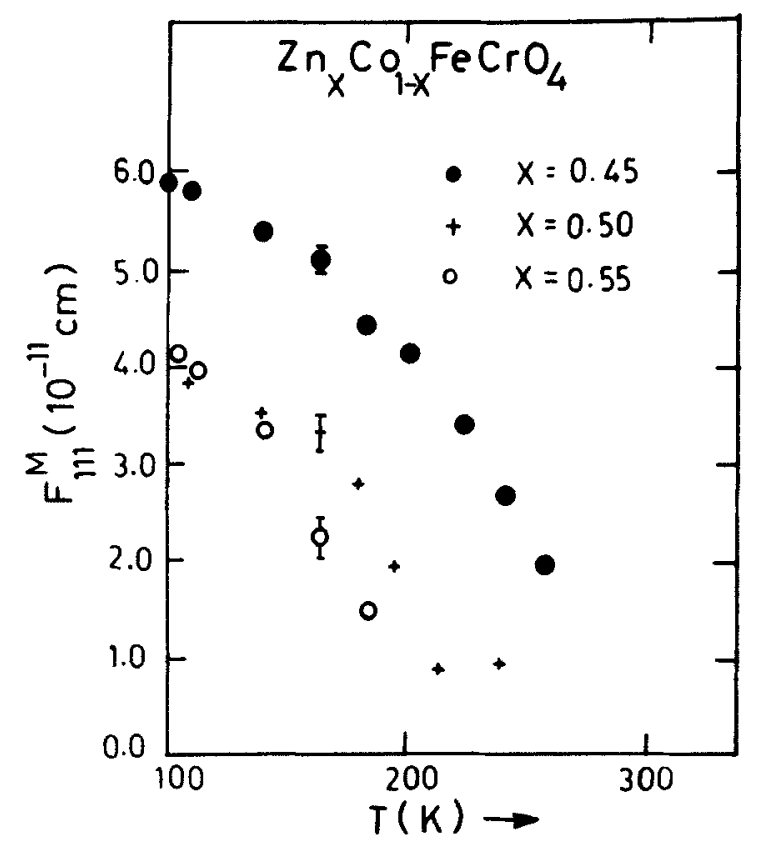

Figure 11. Temperature dependence of the (111) magnetic structure factor in $\mathrm{Zn}_{x} \mathrm{Co}_{1-x} \mathrm{FeCrO}_{4}$.

measured in the temperature range $320>T>20 \mathrm{~K}$. As remarked earlier, the magnetic contributions to the Bragg intensities in the $(220)$ reflection stems from the ordered $A$ site moment alone. Likewise, for the (222) reflection, only the $B$ site ordered moment contributes. These plots (figures 11 and 12 ) clearly show that the temperature dependence of the site moments do not follow the smooth trend one expects for a conventional ferrimagnet for $x>0.50$ in the temperature range $300>T>15 \mathrm{~K}$.

The site moments at $100 \mathrm{~K}$ are summarized in table 1 . Since the mixed spinel under study is ionic, to a very good approximation one can estimate the free ion site moments in this system, taking into account the cation distribution and the ionic states of the magnetic ions. In such spinels it is known that the cobalt and chromium ions are invariably in the divalent and trivalent states respectively. Iron is generally expected to be in the trivalent state which was confirmed further by our Mössbauer patterns taken on these compositions at room temperature. The estimated values based on the free-ion site moments consistent with the observed cation distribution are summarized in table 1 for comparison. In the spinel structure, if the magnetic ions are all ordered collinearly (Neel ordering), then one expects the ordered site moments deduced from normal Bragg reflections such as (111), (220), (222) and (331) to be close to the estimated free ion moments since, in such a situation, the moments of the magnetic ions will be fully aligned along the longitudinal direction (i.e. along the axis of broken symmetry). On the other hand, if the magnetic ordering is noncollinear, the ordered site moments deduced from the normal Bragg reflections will correspond only to the longitudinal components since the transverse components give rise to the (200) Bragg reflection which is purely magnetic. We shall touch upon this aspect later. The site moments derived from the Bragg reflections (table 1) therefore correspond to the longitudinal components of the ordered moment. It is seen that while for 


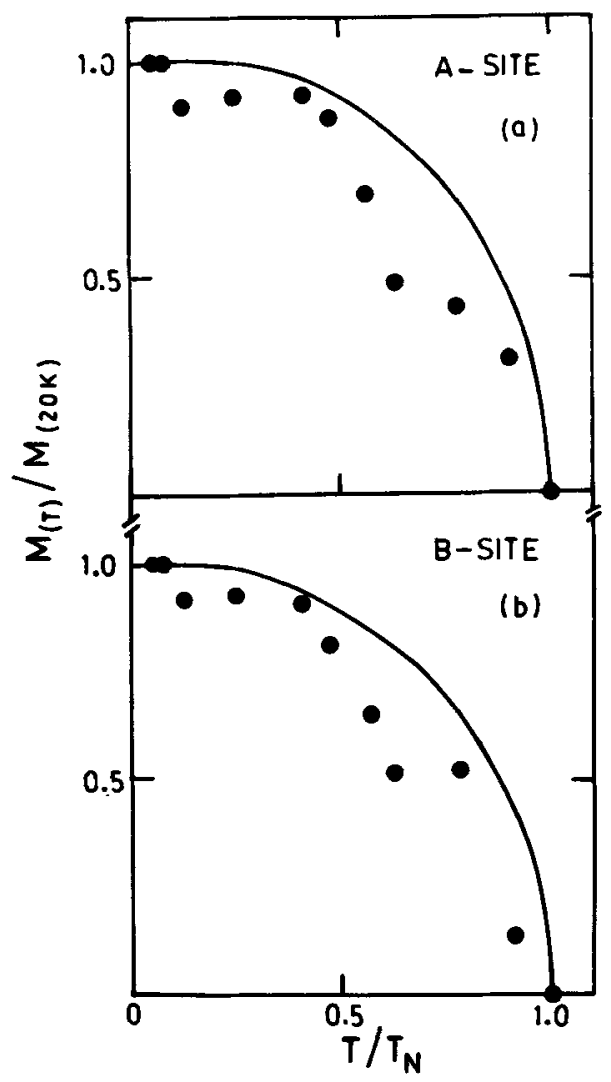

Figure 12. Temperature dependence of the magnetic structure factor for (a) the (220) reflection ( $A$ site) and (b) the (222) reflection ( $B$ site). The error in measurement is indicated by the size of the dots. Solid curves are the Brillouin functions.

$x=0.45$ the $A$-site moment is close to the free ion value, it is about $20 \%$ less for $x>0.45$. The $B$ site moments, on the other hand, are substantially smaller than their estimated free-ion values suggesting that the $B$-site moments are highly non-collinear.

A plausible explanation for the loss in the $A$ site ordered moment observed for $x>0.45$ is that, coexisting with the infinite magnetic network, there are finite clusters which are essentially disordered and do not contribute to the Bragg intensities. The reason we surmise for the existence of disordered finite clusters and not individual paramagnetic spins is that the latter case should give rise to a $Q$-dependent paramagnetic neutron background at low angles which we do not see in any of our diffraction patterns (finite clusters will also give rise to a $Q$-dependent disordered scattering which is observable only at very small angles). Indeed, for the $x=0.50$ specimen, as we approach $20 \mathrm{~K}$ we are able to recover to a great extent the expected $A$-site ordered moment (the $B$ site ordered moment at $20 \mathrm{~K}$ still being $1.7 \mu_{B}$ only), indicating that, as one lowers the temperature, most of these clusters progressively align themselves in phase with the infinite network.

We had remarked earlier that the $B$-site moments are highly non-collinear for all three compositions and that spatial ordering of the transverse spin components should 


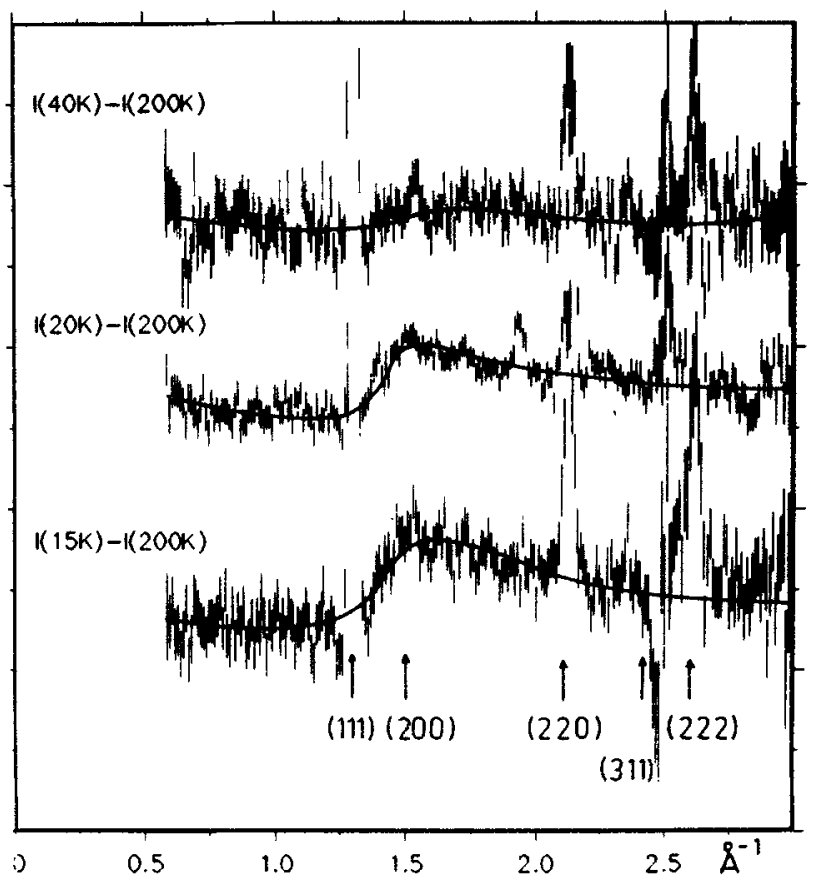

Figure 13. Difference intensity patterns for three temperatures for $x=0-50$, showing the build-up of the diffuse hump at $20 \mathrm{~K}$.

give rise to the (200) Bragg reflection which is purely magnetic in nature. The magnetic structure factor for this reflection is expected to be strong since the transverse components of the $B$-site moments are nearly twice as large as the longitudinal components. Nevertheless, this reflection was not seen in the diffraction patterns of any of the samples down to $100 \mathrm{~K}$ indicating that the transverse components do not contribute to the ordered moment as seen by neutrons.

For the $x=0.50$ sample, the neutron diffraction measurements were extended to $15 \mathrm{~K}$ to check the nature of the transition seen around $20 \mathrm{~K}$ in the $\chi_{\mathrm{ac}}$ for $x \geqslant 0.50$. Figure 13 shows the difference intensity patterns in the low- $Q$ region for three different temperatures $(40,20$ and $15 \mathrm{~K})$ with the $200 \mathrm{~K}$ intensity pattern as the base. It is clearly seen, that around $20 \mathrm{~K}$, a broad diffuse hump appears around $Q=1.5 \AA^{-1}$ (the $Q$ value corresponding to the (200) Bragg reflection). This is an unambiguous signature of the build-up of short-range correlations of the transverse magnetic components in the system. The transverse components of the $B$-site moments are in the fully disordered state down to $20 \mathrm{~K}$ and around this temperature they freeze to give rise to short-range order.

While measuring the temperature evolution of the (111) Bragg intensity in the $x=0.50$ specimen on the POLARIS instrument in the temperature region $100-6 \mathrm{~K}$, a very unexpected feature was observed (see figure 14). The intensity remains fairly constant $100 \mathrm{~K}$ downwards, goes through a slight maximum at around $30 \mathrm{~K}$, then remains fairly constant down to $15 \mathrm{~K}$ and unexpectedly and sharply decreases thereafter. Nevertheless, the broad hump in the $Q$ region of $1.5 \AA^{-1}$ persists as shown in the inset to figure 14. 


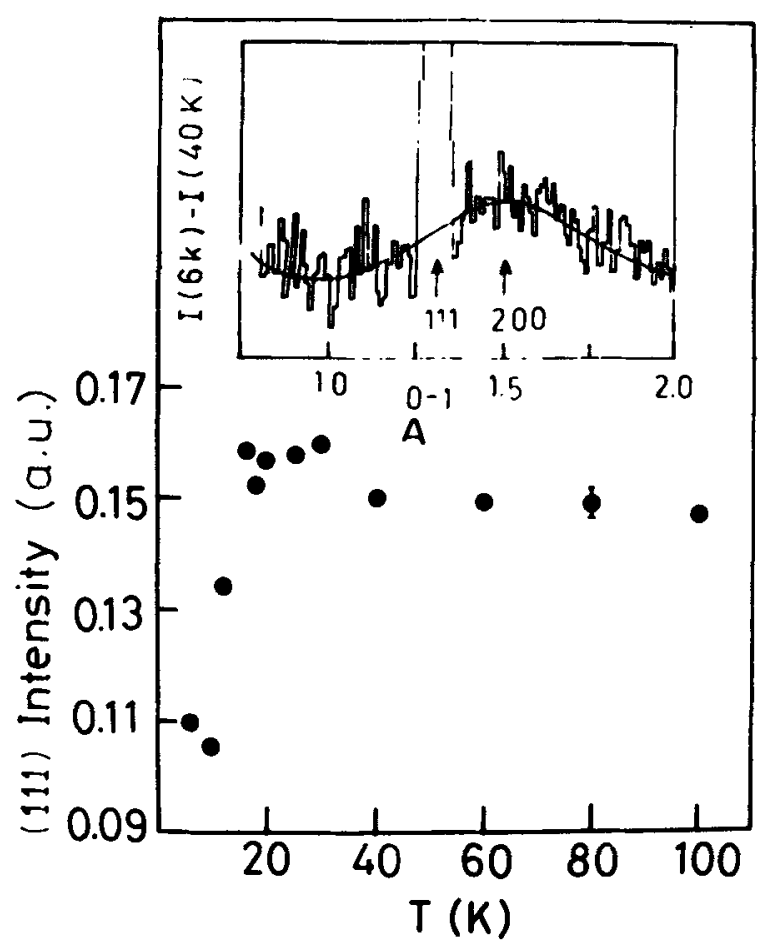

Figure 14. Temperature dependence of the intensity of the (111) Bragg reflection down to $6 \mathrm{~K}$. The inset shows the difference intensity pattern indicating the persistence of the diffuse hump down to $6 \mathrm{~K}$.

\section{Discussion}

Given the complicated two-sublattice spinel structure populated by three different magnetic ion species with differing strengths of exchange bonds, a quantitative interpretation of the experimental features described in the preceding section within the framework of existing theoretical models is an impossible task. What can be attempted is a phenomenological explanation of our observations.

We recall that the range of magnetic dilution in the $A$ sublattice of $\mathrm{Zn}_{x} \mathrm{Co}_{1-x} \mathrm{FeCrO}_{4}$ studied here is around the $A$ site percolation threshold $c_{p}(=0.429)$, with $c-c_{p}=0.12$, 0.07 and 0.02 for $x=0.45,0.50$ and 0.55 respectively. A consequence of magnetic dilution near $c_{p}$ is to create, on a microscopic scale, magnetic inhomogeneities arising out of magnetic atom concentration fluctuations which, in principle, can lead to the formation of finite clusters coexisting with the infinite magnetic network. Moreover, topological frustration is an inherent feature of a spinel lattice. In the absence of magnetic dilution, the intersublattice exchange $J_{A B}$ predominates to establish conventional long-range magnetic ordering. Effects of frustration get accentuated with progressive magnetic dilution of either sublattice which can give rise to perturbed ordered states.

We had remarked earlier that in the spinel lattice the presence of long-range magnetic ordering of the longitudinal spin components is seen in the magnetic contributions to the Bragg intensities of the normal reflections while the presence of long-range magnetic ordering of the transverse spin components is seen in the appearance of 
the purely magnetic (200) Bragg reflection. For the $x=0.50$ specimen the latter reflection is completely absent right down to $20 \mathrm{~K}$, below which temperature a broad hump is seen around the (200) Bragg position (see figure 13). This broad hump persists down to $6 \mathrm{~K}$. Recalling the perturbed ordered phases theoretically predicted by various workers, we can say that we have observed the URF phase in the region $20 \mathrm{~K}<T<T_{N}$ and the CRF or semi-spin-glass phase in the region $T<20 \mathrm{~K}$. The shoulder in the $\chi_{\text {ac }}$ curve for $x=0.50$ (figure 8 ) around $20 \mathrm{~K}$ seems to indicate the transition from the URF to the CRF phase. For the $x=0.55$ specimen, our neutron data taken at different temperatures down to $100 \mathrm{~K}$ indicate that, over this temperature range, this specimen is also in the URF phase. From the strong similarity of the $\chi_{\mathrm{ac}}$ curves for the $x=0.50$ and 0.55 compositions, including the shoulder around $20 \mathrm{~K}$, we surmise that the $x=0.55$ specimen also suffers a transition from the URF to the CRF phase around $20 \mathrm{~K}$. For the $x=0.45$ composition, on the other hand, both the neutron and the susceptibility data indicate its behaviour to be different from the other two compositions. The neutron data show the existence of the URF phase for $T_{N}>T>100 \mathrm{~K}$ and presumably continue to be in the same phase for $T<100 \mathrm{~K}$ as borne out by the rather flat $\chi_{\mathrm{ac}}$ response below $100 \mathrm{~K}$.

Close to percolation for $c>c_{p}$, the system is made up of an infinite magnetic network together with a set of finite clusters. Contributions to the neutron Bragg profiles come from the infinite network while the finite clusters can give rise to diffuse scattering. This diffuse scattering will be observable only at very small $Q$ 's owing to the sharp form factor associated with the finite cluster size. In the $Q$ range covered by our neutron data, no diffuse scattering was observed. Nevertheless, these finite clusters seem to influence the Bragg intensities in a rather subtle manner. First, the rather unexpected decrease in the (111) Bragg intensity is observed to occur below $15 \mathrm{~K}$ in the $x=0.50$ specimen along with the persistence of the broad hump at $Q=1.5 \AA^{-1}$ (figure 14). We have no convincing microscopic explanation for this observation. Qualitatively, this can arise when some of the finite clusters which are initially randomly oriented vis-a-vis the infinite network gradually freeze as the temperature is lowered into a configuration where they are preferably oriented out of phase with the infinite network, thus provoking a decrease in the Bragg intensity. The non-Brillouin-like temperature dependence of the ordered moment, especially for the $x=0.50$ and 0.55 compositions (figures 11 and 12) can also be qualitatively understood in a similar picture of an inhomogeneous system consisting of an infinite network and finite clusters. As one lowers the temperature, some of these clusters lock in phase with the infinite network to enhance the Bragg intensities, some lock themselves out of phase with the infinite network to bring about steps in the temperature response of the Bragg intensity. However, we have no clue as to the microscopic mechanism which can give rise to such a complex behaviour.

The broad peaks in the ac susceptibility curves around $140 \mathrm{~K}$ (for $x=0.50$ and 0.55 ) and around $280 \mathrm{~K}$ (for $x=0.45$ ) which mimic a spin glass transition may, in reality, indicate either a progressive freezing of these finite clusters or alternatively may reflect a domain-wall movement and pinning.

\section{Conclusion}

The exotic magnetic phases we have seen in $\mathrm{Zn}_{x} \mathrm{Co}_{1-x} \mathrm{FeCrO}_{4}$ using the neutron diffraction technique are perhaps more complex than the URF and the CRF phases 
predicted by Mookerjee (1980) given the existence of finite clusters and their influence on the infinite magnetic network.

We had also studied (Chakravarthy et al 1991) by neutron diffraction and magnetic susceptibility techniques the disordered spinel, $\mathrm{Zn}_{0.5} \mathrm{Co}_{0.5} \mathrm{Fe}_{2-y} \mathrm{Cr}_{y} \mathrm{O}_{4}(y=0.9$ and $1 \cdot 1)$, to see how the relative concentrations of $\mathrm{Fe}$ and $\mathrm{Cr}$ on the octahedral sites influence the magnetic phase. Comparison with our study on $\mathrm{Zn}_{x} \mathrm{Co}_{1-x} \mathrm{FeCrO}_{4}$ shows that the effect of a slight increase in the magnetic $\mathrm{Cr}$-ion concentration at the $B$ site is similar to that produced by an increase of diamagnetic $\mathrm{Zn}$-ion concentration at the $A$ site.

In our view, this fascinating study of the exotic magnetic phases in these mixed spinels is not complete. We need to seek answers to the following questions: What are the sizes of these finite clusters and how do they influence the ordering of the infinite network? Small-angle neutron scattering studies need to be taken up to answer these questions, and to determine also the relative scales of the correlation lengths associated with the finite clusters and the infinite network. These studies can be taken up at Dhruva on the small angle neutron scattering spectrometer that will be installed on one of the cold neutron guides.

\section{Acknowledgements}

This is essentially a review of the work done by our Trombay group (Madhav Rao 1989; Chakravarthy et al 1991). It is a pleasure to acknowledge the collaboration of my colleagues R Chakravarthy, S K Paranjpe, S K Kulshreshtha and S B Roy in these studies. I wish to thank the Materials Research Society of India for having given me the opportunity to present this work at its 1993 Annual General Meeting.

\section{References}

Chakravarthy R, Madhav Rao L, Paranjpe S K, Krishna P S R, Kulshreshtha S K and Balakrishnan G 1991 Physica B174 47

Chakravarthy R, Madhav Rao L, Paranjpe S K, Kulshreshtha S K and Roy S B 1991 Phys. Rev. B43 6031

Fiorani D, Gastaldi L, Lapiccirella A, Viticoli S and Tomassini N 1979 Solid State Commun. 32831

Gabay M and Toulouse G T 1981 Phys. Rev. Lett. 47201

Hamedoun M, Wiedemann A, Dorman J L, Nogues M and Rossat-Mignod J 1986 J. Phys. C19 1783, 1803

Lyons D, Kaplan T, Dwight K and Menyuk N 1962 Phys. Rev. 126540

Madhav Rao L. 1985 Bull. Mater. Sci. 7303

Madhav Rao L 1989 Advances in ferrites: Proc. fifth Int. Conf., (New Delhi: Oxford and IBH Publishing Co.) Vol. 1, pp 385-398

Mookerjee A 1980 Pramana-J. Phys. 1411

Muraleedharan K. Srivastava J K, Marathe U R and Vijayaraghavan R 1985 I. Magn. Magn. Mater. 5033

Poole C P and Farach H A 1982 Z. Phys. (Germany) B47 55

Radhakrishnan N K, Paranjpe S K, Madhav Rao L and Satya Murthy N S 1972 Nucl. Phys. Solid State Phys. (India) 146645

Satya Murthy N S, Natera M G, Youser S I, Begum R J and Srivastava C M 1969 Phys. Rev. 181969

Scholl F and Binder K 1980 Z. Phys. (Germany) B39 239

Villain J 1979 Z. Phys. Paris B33 31

Yafet Y and Kittel C 1952 Phys. Rev. 87290 